\author{
Gisela M. Terwindt \\ Michel D. Ferrari \\ Lenore J. Launer
}

\section{The impact of headache on quality of life}

G.M. Terwindt $(\bowtie)$ • M.D. Ferrari

Department of Neurology,

Leiden University Medical Center,

P.O. Box 9600, 2300 RC Leiden,

The Netherlands

e-mail: G.M.Terwindt@lumc.nl

Tel.: +31-71-5262587

Fax: +31-71-5248253

\section{L.J. Launer}

Neuroepidemiology Section,

Laboratory of Epidemiology, Demography, and Biometry,

National Institute on Aging,

Bethesda, Maryland, USA

\begin{abstract}
Population-based studies have shown that migraineurs have reduced HRQoL independent of depression. Furthermore, HRQoL is reduced with increasing migraine attack frequency. In both populationbased and clinic-based studies, CDH patients have reduced HRQoL, most marked in subjects with analgesic overuse. In one population-based study, no difference in HRQoL was found between patients with transformed migraine (TM) and patients with CTTH. However, the number of patients in this study was relatively small. In a clinic-based study, SF-36
\end{abstract}

scores in patients with chronic daily headache $(\mathrm{CDH})$ depended on the percentage of the type of patients in the sample: the greater the proportion of patientsa with TM, the more impaired the SF-36 scores.

Key words HRQoL • Quality of life - Headache • Migraine • Cluster headache

\section{Introduction}

Both general and disease-specific quality of life measurements can be used to evaluate health-related quality of life (HRQoL) in headache patients. General HRQoL measurements provide a method to compare the burden of different types of headache. Furthermore, with a general instrument, the impact on HRQoL of headache patients can be compared with that of other diseases. Examples of general instruments are the Medical Outcomes Study short form-20 (SF-20) and the Medical Outcomes Study short form health survey (SF36). Disease-specific HRQoL questionnaires focus on particular limitations or restrictions associated with a specific disease status and can be useful as an outcome measure in clinical trials or clinical practice to identify treatments which optimise outcomes [1, 2]. Examples of migraine-specific HRQoL instruments are the 24-hour migraine quality of life (24-h MQoL), the migraine-specific quality of life (MSQoL) questionnaire, and the migraine-specific quality of life (MSQ) questionnaire.

HRQoL studies can be performed in patients referred to a headache clinic but these patient groups represent a selected group of headache patients. This may not be a problem when studying the effect of specific headache treatments on HRQoL. Population-based studies also identify patients with headache who never see a physician for their headache and, therefore, will give a clearer picture of the effect of headache on HRQoL.

\section{Impact of migraine on HRQoL in the general population}

In a large Dutch population-based study, we compared the HRQoL of migraineurs and non-migraineurs [3]. HRQoL of 
migraineurs was also compared to that of people from the same cohort with asthma, chronic musculoskeletal pain, or both conditions. These conditions reportedly have a higher prevalence in migraineurs than in non-migraineurs [4-9] and both affect HRQoL [10-13]. In addition, each condition shares different common characteristics with migraine: asthma is a chronic paroxysmal disorder and chronic musculoskeletal pain is a chronic pain disorder.

To assess HRQoL, we used the Dutch version of the RAND-36, a questionnaire developed from the Medical Outcomes Study general health survey instrument [14]. The RAND-36 includes 36 items measuring health across eight domains (Table 1). Responses to each item within a dimension are combined to generate a score from 0-100, where 100 indicates best functioning [14]. Because the RAND-36 measures the perception of health in the previous four weeks, the analysis was based on 620 migraineurs who had active migraine during the last year.
Of the 620 migraineurs who were identified, 396 persons (63.9\%) had migraine without aura only, 111 (17.9\%) had migraine with aura, and $81(13.1 \%)$ had attacks of both types; $5 \%$ could not be classified. The median number of migraine attacks was 12 per year; $25 \%$ of migraineurs suffered at least two attacks per month. Among the nonmigraineurs, 171 persons $(3.2 \%)$ were identified with asthma and 1345 persons (25\%) with chronic musculoskeletal pain. Among the migraineurs, 30 persons (5\%) had asthma and 239 persons (39\%) had chronic musculoskeletal pain. Compared to non-migraineurs, migraineurs had significantly more frequent asthma $(\mathrm{OR}=1.6 ; 95 \% \mathrm{CI}, 1.1-2.4)$ and chronic musculoskeletal pain $(\mathrm{OR}=1.7 ; 95 \% \mathrm{CI}, 1.5-2.1)$.

The scores of the eight RAND-36 domains for migraineurs are shown in Fig. 1. Compared to nonmigraineurs, migraineurs reported diminished functioning on all eight RAND-36 dimensions which cover physical, social, and mental functioning $(p<0.0001)$. There was no

Table 1 Dimensions of HRQoL in the Medical Outcomes Study short form RAND-36

\begin{tabular}{lrl}
\hline Dimensions & Items, $\mathrm{n}$ & Definition \\
\hline Physical functioning (PF) & 10 & Capacity to perform a variety of common physical activities \\
Social functioning (SF) & 2 & Extent to which health interferes with social activities (previous 4 weeks) \\
Role limitations, physical (RP) & 4 & Extent to which physical health interferes with daily activities (previous 4 weeks) \\
Role limitations, emotional (RE) & 3 & Extent to which emotional problems interfere with daily activities (previous 4 weeks) \\
Mental health (MH) & 5 & General mood or affect in previous 4 weeks \\
Bodily pain (BP) & 2 & Bodily pain in previous 4 weeks \\
Vitality (VT) & 4 & General energy, level of fatigue in previous 4 weeks \\
General health perception $(\mathrm{GH})$ & 5 & Overall rating of health in general \\
\hline
\end{tabular}

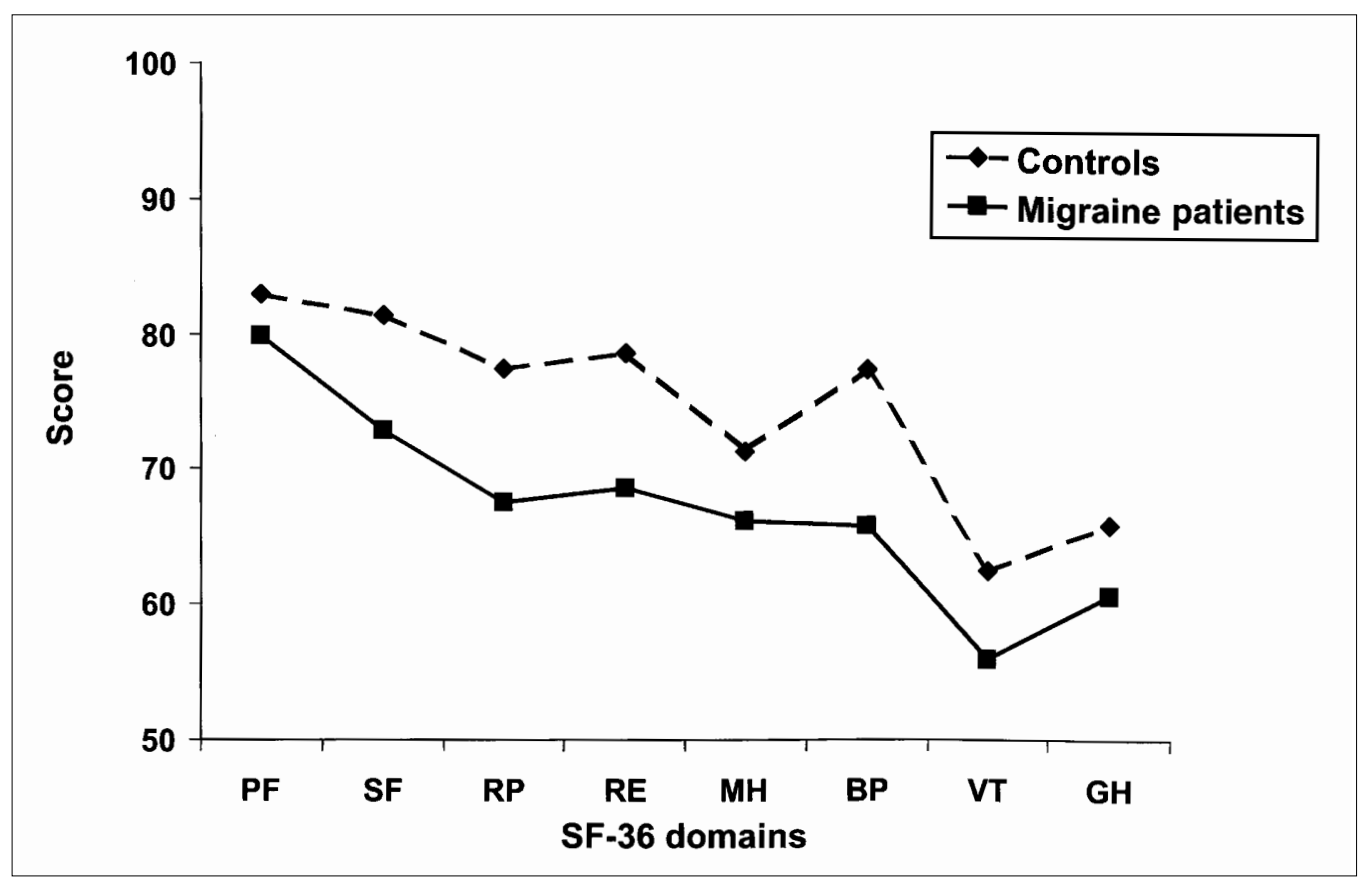

Fig. 1 RAND-36 scores in patients with migraine $(\mathrm{n}=620)$ and in controls $(\mathrm{n}=5378)$. A lower score indicates diminished functioning or well-being. There is a significant difference between migraineurs and controls $(p<0.0001)$, controlling for sex, age, socioeconomic class, region of residence, and marital status for all domains. 
difference in HRQoL among migraine subtypes. For all eight dimensions of the RAND-36, HRQoL decreased with increasing frequency of migraine attacks $(p<0.0002)$.

The scores of the eight RAND-36 domains for subjects with migraine, asthma and both asthma and migraine are shown in Fig. 2. Compared to controls, asthma cases scored lower on three RAND-36 dimensions, including physical functioning, pain and general health perception. Compared to asthma cases, migraineurs scored significantly lower on five of the eight RAND-36 domains, including social functioning, role limitations (emotional), mental health, pain, and vitality. There was no difference in the physical functioning domains and the general health perception domain between migraineurs and asthma cases. Compared to controls, those with both asthma and migraine had significantly lower scores on the dimensions including physical functioning, role limitations (physical), mental health, pain, vitality, and general health perception. Compared to those with migraine only, cases with both migraine and asthma had significantly lower scores on dimensions of physical functioning and general health perception. Compared to those with asthma only, cases with both migraine and asthma had significantly lower scores on the dimension of pain and general health perception.

The RAND-36 scores for migraineurs, subjects with chronic musculoskeletal pain and subjects with both chronic musculoskeletal pain and migraine are shown in Fig. 3. Compared to controls, persons with chronic musculoskeletal pain scored significantly lower on all RAND-36 dimensions. Compared to migraineurs, those with chronic musculoskeletal pain scored significantly lower on five of the eight RAND-36 domains, including physical functioning, role limitations (physical), pain, vitality, and general health perception. Compared to controls, persons with both chronic musculoskeletal pain and migraine scored significantly lower on all RAND-36 dimensions. Compared to subjects with only migraine or chronic musculoskeletal pain, those with both migraine and chronic musculoskeletal pain had significantly lower scores for all eight RAND-36 domains.

In this population-based study, we found that migraineurs, regardless of subtype, had poorer HRQoL compared to non-migraineurs. Frequency of attacks (used as a measure of disease severity) showed a clear inverse rela-

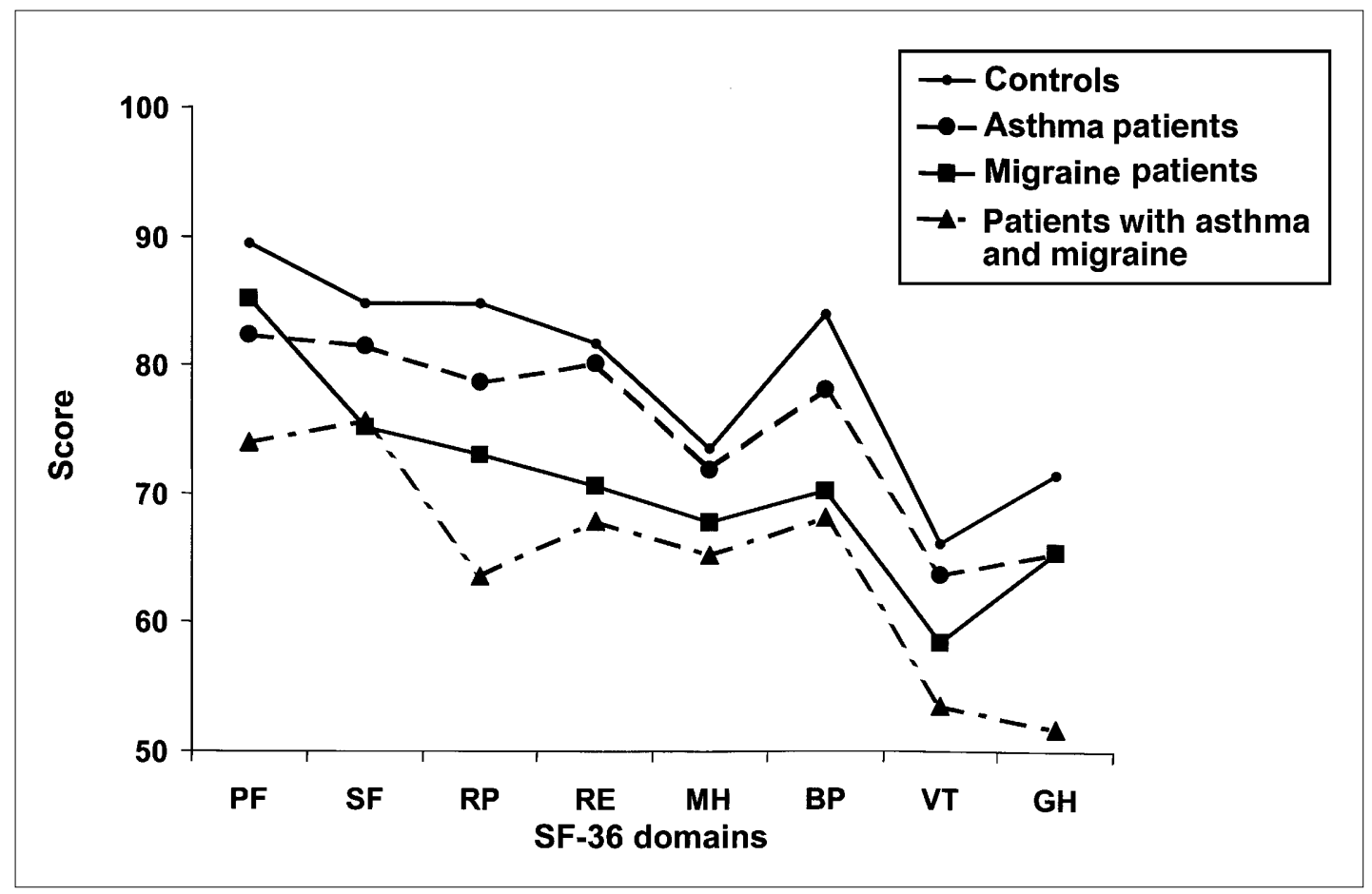

Fig. 2 RAND-36 scores in patients with migraine $(n=589)$, asthma $(n=171)$ or both migraine and asthma $(n=30)$, and in controls $(n=5293)$. Fifteen persons are not included in this figure because of missing data on asthma. A lower score indicates diminished functioning or wellbeing. Analysis of variance controlling for sex, age, socio-economic class, region of residence, and marital status revealed significant differences between: asthma patients and controls for the PF, BP, GH domains $(p<0.0001$ to $p<0.0003)$; migraine patients and controls for all domains $(p<0.0001)$; patients with both migraine and asthma vs. controls for the PF, RP, MH, BP, VT, GH domains $(p<0.0001$ to $p<0.0038)$; asthma patients and migraine patients for the SF, RE, MH, BP, VT domains $(p<0.0006$ to $p<0.0030)$; asthma patients and patients with both asthma and migraine for the BP and GH domains $(p<0.0028)$; migraine patients and patients with both migraine and asthma for the PF and GH domains $(p<0.0002)$. Abbrevations are defined in Table 1 


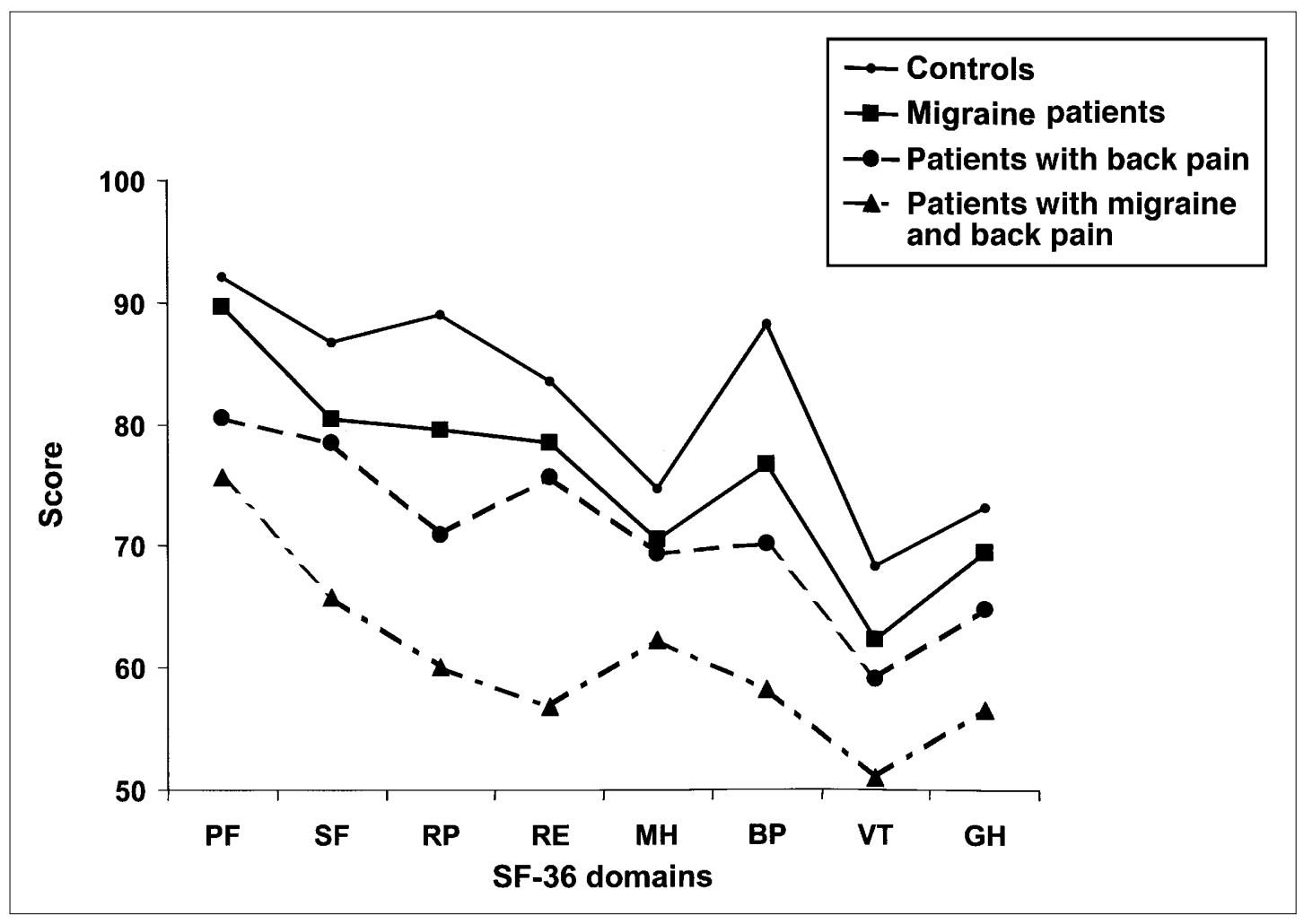

Fig. 3 RAND-36 scores in patients with migraine with $(n=381)$, chronic musculoskeletal pain (back pain) ( $n=1345)$ or both migraine and chronic musculoskeletal pain $(n=239)$, and in controls $(n=4033)$. A lower score indicates diminished functioning or well-being. Analysis of variance controlling for sex, age, socio-economic class, region of residence, and marital status revealed significant differences between: patients with chronic back pain and controls for all RAND-36 domains $(p<0.0001)$; migraine patients and controls for all RAND-36 domains $(p<0.0001$ to $p<0.0042)$; patients with both chronic back pain and migraine vs. controls for all RAND-36 domains $(p<0.0001)$; patients with chronic back pain and migraine patients for the PF, RP, BP, VT, GH domains ( $p<0.0001$ to $p<0.0007)$; patients with chronic back pain and patients with both migraine and chronic back pain for all RAND-36 domains $(p<0.0001)$; and migraine patients and patients with both conditions for all RAND-36 domains $(p<0.0001)$. Abbrevations are defined in Table 1

tionship, indicating that HRQoL reduced with increasing migraine attack frequency.

In sub-analyses, we compared migraine with asthma and chronic musculoskeletal pain for HRQoL and found that migraineurs had poorer HRQoL compared to patients with asthma, but not compared to patients with chronic musculoskeletal pain. When comparing these three disorders, we found that the impact of migraine was greater on social and mental functioning, whereas asthma and chronic musculoskeletal pain had more impact on physical functioning. Comorbidity of migraine with asthma or chronic musculoskeletal pain resulted in lower social, mental, and physical functioning than one of the two conditions alone. Possibly, migraineurs have a lower pain threshold. However, we can not exclude the possibility of reporting bias resulting from more subjects with migraine self-reporting chronic musculoskeletal pain or asthma.

From this study, we concluded that migraineurs, regardless of subtype, suffer from compromised HRQoL, especial- ly those with high frequency of attacks. Migraineurs have a higher prevalence of asthma and chronic musculoskeletal pain, and decreased HRQoL in subjects with migraine may be partly due to these comorbid conditions.

It has been recognised that migraineurs have an increased occurrence of depression $[15,16]$, and depression is associated with poorer HRQoL $[12,17]$. In this study the (co-)occurrence of depression was not investigated and, therefore, the influence of co-morbidity of depression on HRQoL in migraineurs cannot be estimated.

\section{Migraine, quality of life, and depression}

Lipton et al. [18] conducted two population-based studies in the United States and United Kingdom in which 389 migraine cases (with 6 or more attacks per year) and 379 non-migraine controls completed the short form (SF)-12 and 
the primary care evaluation of mental disorders (PRIMEMD) questionnaire, a mental health screening tool. The SF12 measures HRQoL in two domains: a mental health component score (MCS-12) and a physical health component score (PCS-12).

Depression and migraine were highly comorbid; $47 \%$ (183/389) of migraineurs had PRIME-MD defined depression compared to $17 \%$ (64 of 379) of the controls (adjusted prevalence ratio, $2.7 ; 95 \% \mathrm{CI}, 2.1$ to 3.5 ). Depressed subjects had lower HRQoL scores than non-depressed subjects. There was no significant difference in MCS-12 and PCS-12 scores between migraine cases and controls among depressed subjects. There was, however, a significant difference in MCS-12 and PCS-12 scores between non-depressed migraine cases and controls. Both depression and migraine were significantly and independently associated with lower MCS-12 and PCS-12 scores. Depression had a larger effect than migraine on both scores.

Attack frequency was a strong predictor of decreased MCS-12 and PCS-12 scores. For the MCS-12, headacherelated disability (headache interfered with daily activities half the time or more) was also a strong predictor of decreased MCS-12 scores.

Lipton and colleagues concluded from this study [18] that migraineurs have reduced $\mathrm{HRQoL}$ regardless of whether they are also depressed. However, HRQoL was significantly reduced in subjects with migraine and depression compared with those who were not depressed.

Both our study [3] and that of Lipton et al. [18] found that headache frequency was an important predictor of HRQoL.

\section{Does HRQoL differ among headache diagnoses?}

To analyse the differences in HRQoL associated with different headache diagnoses, Solomon et al. [19] performed a clinic-based study of 208 headache patients. Of these 208 patients, 79 patients had migraine, 41 had tension-type headache, 13 had cluster headache, and 49 had mixed headaches (chronic tension-type headache and migraine). The Medical Outcomes Study short form health survey (SF20) was used to assess HRQoL.

Cluster headache patients had a significantly higher (worse) pain score and higher frequency of poor health due to pain than patients with migraine headache. Cluster patients had a generally well preserved physical functioning compared with tension-type or mixed headache patients. Cluster and tension-type headache patients showed more limitations in social functioning than migraine. Chronic tension-type headache patients were marked by a lower level of mental health compared to those with migraine. Solomon et al. [19] concluded that distinct headache diagnoses are marked by unique patterns of impairment of HRQoL. However, the patients sample in this study may represent the more severely afflicted headache patients who seek care at a specialised headache referral center. Furthermore, comorbidity and confounding by depression were not investigated in this study.

\section{HRQoL differences between patients with episodic and transformed migraine}

To investigate specifically whether there are HRQoL differences between patients with episodic migraine and those with transformed migraine, Meletiche et al. [20] investigated 90 patients (46 with transformed migraine, 44 with episodic migraine) with the SF-36 questionnaire [20]. Over the last 90 days prior to their first visit, patient with transformed migraine reported having a headache significantly more often compared with patients with migraine (69 days and 18 days, respectively). Compared with patients with episodic migraine, patients with transformed migraine had significantly lower mean scores on seven of the eight SF-36 domains and both the mental and physical summary scores of the SF-36.

Meletiche et al. [20] concluded that patients with transformed migraine had a lower HRQoL than patients with episodic migraine and suggested that headache chronicity associated with transformed migraine had a significant influence on HRQoL. Comorbidity and confounding by depression were not investigated in this study.

\section{HRQoL differs among headache diagnoses}

Wang et al. [21] evaluated HRQoL in 901 patients who visited a headache clinic by using the Medical Outcomes Study short form health survey (SF-36) and the hospital anxiety and depression scale (HADS). They compared HRQoL in 193 patients with episodic migraine and in 541 patients with chronic daily headache $(\mathrm{CDH})$. Of the $\mathrm{CDH}$ group, 310 patients were classified as having transformed migraine (TM) and 231 patients were considered to have chronic tension-type headache (CTTH).

SF-36 scores were compared after controlling for the HADS score, age, gender, education, and chronic illness by multiple linear regression analyses. Compared with migraine patients, patients with TM had significantly lower scores on all scales, except for the PF, SF and RE scales. Patients with CTTH had compatible SF-36 scores in comparison with patients with migraine, except for $\mathrm{BP}(\mathrm{CTTH}>$ 
migraine) and $\mathrm{MH}$ (CTTH > migraine) scales. Compared with CTTH patients, TM patients scored significantly lower on all SF-36 scales except for PF and RE. Wang et al. [21] concluded that the SF-36 scores in patients with CDH depended on the percentage of the type of patients in the sample: the greater the proportion of TM patients, the more impaired the SF-36 scores were.

\section{Quality of life in chronic daily headache}

Guitera et al. [22] studied HRQoL by SF-36 in 89 unselected subjects from an epidemiological study in the general population who suffered from chronic daily headache (CDH) and compared HRQoL in this group with that of 89 healthy matched subjects. Of the $\mathrm{CDH}$ patients, 50 subjects suffered from chronic tension-type headache (CTTH), 37 had transformed migraine (TM), and two had new daily persistent headache. HRQoL of TM patients was also compared to that in patients with episodic migraine (EM). $\mathrm{CDH}$ with analgesic overuse was diagnosed in 22 patients. CDH subjects showed a significant decrease of all SF-36 domains as compared with healthy controls. There was no significant difference in SF-36 scores in CTTH patients as compared with TM subjects. CDH subjects without analgesic overuse had significant lower scores for physical functioning (PF) and bodily pain (BP) on the SF-36 than those with analgesic overuse. No difference was found for HRQoL between TM subjects and subjects with episodic migraine (EM). Although this study included non-selected subjects from a population-based sample, the number of patients was small and comorbidity with depression was not investigated.

\section{Conclusions}

Population-based studies have shown that migraineurs have reduced HRQoL independent of depression. Furthermore, HRQoL is reduced with increasing migraine attack frequency. In both population-based and clinic-based studies, $\mathrm{CDH}$ patients have reduced HRQoL, most marked in subjects with analgesic overuse. In one population-based study, no difference in HRQoL was found between patients with transformed migraine (TM) and patients with CTTH. However, the number of patients in this study was relatively small. In a clinic-based study, SF-36 scores in patients with chronic daily headache $(\mathrm{CDH})$ depended on the percentage of the type of patients in the sample: the greater the proportion of patients with TM, the more impaired the SF36 scores.

\section{References}

1. Lipton RB, Stewart WF (1995) Healthrelated quality of life in headache research. Headache 35(8):447-448

2. Solomon GD, Santanello N (2000) Impact of migraine and migraine therapy on productivity and quality of life. Neurology 55[9 Suppl 2]:S29-S35

3. Terwindt GM, Ferrari MD, Tijhuis M, Groenen SM, Picavet HS, Launer LJ (2000) The impact of migraine on quality of life in the general population: the GEM Study. Neurology 55(5):624-629

4. Chen TC, Leviton A (1990) Asthma and eczema in children born to women with migraine. Arch Neurol 47:1227-1230

5. Duckro PN, Schultz KT, Chibnall JT (1994) Migraine as a sequela to chronic low back pain. Headache 34:279-281

6. Schéle R, Ahlborg B, Ekbom K (1978) Physical characteristics and allergic history in young men with migraine and other headaches. Headache 18:80-86
7. Stang P, Sternfeld B, Sidney S (1996) Migraine headache in a prepaid health plan: ascertainment, demographics, physiological, and behavioral factors. Headache 36:69-76

8. Ziegler DK, Hassanein RS, Couch JR (1977) Characteristics of life headache histories in a nonclinic population. Neurology 27:265-269

9. Vernon H, Steiman I, Hagino C (1992) Cervicogenic dysfunction in muscle contraction headache and migraine: a descriptive study. J Manipulative Physiol Ther 15(7):418-429

10. Solomon GD, Skobieranda FG, Gragg LA (1993) Quality of life and wellbeing of headache patients: measurement by the Medical Outcome Study instrument. Headache 33:351-358

11. Stewart AL, Greenfield S, Hays RD, Wells K, Rogers WH, Berry SD, McGlynn EA, Ware JE (1989) Functional status and well-being of patients with chronic conditions. JAMA 262:907-913
12. Lyons RA, Lo SV, Littlepage BNC (1994) Comparative health status of patients with 11 common illnesses in Wales. J Epidemiol Commun Health 48:388-390

13. Beltman FW, Heesen WF, Tuinman RG, Meyboom-de Jong B (1995) Functionele status van patienten met chronische aandoeningen. Tijdschr Soc Gezondheidsz 73:128-134

14. van der Zee K, Sanderman R, Heyink J (1996) A comparison of two multidimensial measures of health status: the Nottingham health profile and the RAND 36-item health survey 1.0. Quality Life Res 5:165-174

15. Breslau N, Davis GC (1993) Migraine, physical health and psychiatric disorder: a prospective epidemiologic study in young adults. J Psychiatr Res 27:211-221

16. Keck PE, Merikangas KR, McElroy SL, Strakowski SM (1994) Diagnostic and treatment implications of psychiatric comorbidity with migraine. Ann Clin Psychiatry 6:165-71 
17. Wells KB, Stewart A, Hays RD, Burnam MA, Rogers W, Daniels M, Berry S, Greenfield S, Ware J (1989) The functioning and well-being of depressed patients. Results from the medical outcomes study. JAMA 262:914-919

18. Lipton RB, Hamelsky SW, Kolodner KB, Steiner TJ, Stewart WF (2000) Migraine, quality of life, and depression: a population-based case- control study. Neurology 55(5):629-635
19. Solomon GD, Skobieranda FG, Gragg LA (1994) Does quality of life differ among headache diagnoses? Analysis using the Medical Outcomes Study instrument. Headache 34(3):143-147

20. Meletiche DM, Lofland JH, Young WB (2001) Quality-of-life differences between patients with episodic and transformed migraine. Headache 41(6):573-578
21. Wang SJ, Fuh JL, Lu SR, Juang KD (2001) Quality of life differs among headache diagnoses: analysis of SF-36 survey in 901 headache patients. Pain 89(2-3):285-292

22. Guitera V, Munoz P, Castollo J, Pascual J (2002) Quality of life in chronic daily headache. Neurology 58:1062-1065 